\title{
Propofol alleviates hypoxia-induced nerve injury in PC-12 cells by up-regulation of microRNA-153
}

\author{
Mingwei He, Haiyan Sun, Jinlei Pang, Xiangfei Guo, Yansong Huo, Xianhong Wu, Yaguang Liu and Jun Ma* (D)
}

\begin{abstract}
Background: Although the neuroprotective role of propofol has been identified recently, the regulatory mechanism associated with microRNAs (miRNAs/miRs) in neuronal cells remains to be poorly understood. We aimed to explore the regulatory mechanism of propofol in hypoxia-injured rat pheochromocytoma (PC-12) cells.

Methods: PC-12 cells were exposed to hypoxia, and cell viability and apoptosis were assessed by CCK-8 assay and flow cytometry assay/Western blot analysis, respectively. Effects of propofol on hypoxia-injured cells were measured, and the expression of miR-153 was determined by stem-loop RT-PCR. After that, whether propofol affected PC-12 cells under hypoxia via miR-153 was verified, and the downstream protein of miR-153 as well as the involved signaling cascade was finally explored.

Results: Hypoxia-induced decrease of cell viability and increase of apoptosis were attenuated by propofol. Then, we found hypoxia exposure up-regulated miR-153 expression, and the level of miR-153 was further elevated by propofol in hypoxia-injured PC-12 cells. Following experiments showed miR-153 inhibition reversed the effects of propofol on hypoxia-treated PC-12 cells. Afterwards, we found BTG3 expression was negatively regulated by miR-153 expression, and BTG3 overexpression inhibited the mTOR pathway and AMPK activation. Besides, hypoxia inhibited the mTOR pathway and AMPK, and these inhibitory effects could be attenuated by propofol.
\end{abstract}

Conclusion: Propofol protected hypoxia-injured PC-12 cells through miR-153-mediataed down-regulation of BTG3. BTG3 could inhibit the mTOR pathway and AMPK activation.

Keywords: Hypoxia injury, PC-12 cells, Propofol, miR-153, BTG3, mTOR/AMPK

\section{Background}

The brain is critically dependent upon a continuous blood-borne supply of oxygen, since the normal brain metabolism exerts a high demand of oxygen whereas a limited intrinsic stores of high-energy phosphate compounds [1]. Deprivation of enough oxygen in the brain, induced by a clot within an artery, may ultimately lead to stroke [2]. Reported as the fourth leading cause of death in the United States, stroke is a devastating neural event which causes serious long-term disability $[3,4]$. Although the recombinant tissue plasminogen activator (rtPA) has been applied to improve morbidity and mortality of stroke, the therapeutic window is limited [5]. Innovative

\footnotetext{
* Correspondence: majun0083@sina.com

Department of Anesthesiology, Beijing Anzhen Hospital, Capital Medical University, No.2, Anzhen Road, Chaoyang District, Beijing 100029, China
}

neuroprotective strategies for stroke are pressingly needed to improve the prognosis of stroke.

Propofol is a widely used intravenous anesthetic agent with rapid onset and short recovery time [6]. It is an agonist on Gamma-Aminobutyric acid (GABA) receptors, and the tumor suppressive role of propofol as well as the carcinogenesis of propofol has been well documented [7-9]. Recently, accumulating evidence has reported the protective roles of propofol against hypoxia in diverse cell types, including neuronal cells $[10,11]$. For instance, the neuroprotective role of propofol had been verified in hypoxic hippocampal slice [12]. The $\mathrm{CoCl}_{2}$-induced hypoxia injury in mouse hippocampal HT22 cells was attenuated by porpofol through the PP2A/CAMKII $\alpha / n N O S$ pathway [13]. A previous study also proposed that propofol provided neuroprotection to 
injured neuronal cells through inhibiting the Toll-like receptor 4 and nuclear factor $\mathrm{kB}$ signaling pathways [14]. Although the regulatory mechanisms of propofol in neuronal cells have been studied previously, innovative regulatory pathways are waiting to be identified in order to accelerate the application of propofol in the area of neuroprotection.

MicroRNAs (miRNAs/miRs) are small regulatory RNAs which lack protein coding capacity [15]. Despite of the critical regulatory role of miRNAs in cancer progression, the functional roles of miRNAs such as miR-210 and miR-147 in hypoxia-injured neuronal cells have been widely reported $[16,17]$. Although it has been proven that propofol might affect hypoxia-injured cells through regulating miRNAs [18], the regulatory mechanism associated with miRNAs in neuronal cells remains to be poorly understood. MiR-153 is an important tumor regulatory miRNA, acting as either a tumor suppressor or an oncogene in diverse cancer types [19, 20]. Recently, a literature by Liang et al. stated that miR-153 level was up-regulated after hypoxia treatment in breast cancer cells [21]. Kim et al. found miR-153 expression was significantly up-regulated after propofol treatment in adipocyte stem cells, showing approximately 4-fold difference from untreated cells [22]. Additionally, the neuroprotective role of miR-153 has been proven by a previous literature [23]. Those observations described above suggesting that miR-153 might be a downstream miRNA of propofol.

In this study, we constructed in vitro hypoxia cell model using PC-12 cells, and then verified the neuroprotective role of propofol. Furthermore, the possible downstream miRNA of propofol as well as the regulatory axis was also investigated to explain the regulatory mechanism of propofol. Besides, the alteration of involved signaling cascades was also studied.

\section{Methods}

\section{Cell culture and treatments}

The PC-12 cells (ATCC CRL-1721 ${ }^{\mathrm{m}}$ ) were obtained from American Type Culture Collection (ATCC; Manassas, VA, USA). Cells were maintained in RPMI 1640 medium (Hyclone, Logan, UT, USA) containing 10\% heat-inactivated horse serum (Hyclone) and 5\% fetal bovine serum (Hyclone). For incubation under normoxia, cells were grown in a humidified incubator at $37^{\circ} \mathrm{C}$ with $5 \% \mathrm{CO}_{2}$ and $95 \%$ air. For incubation under hypoxia, cells were subjected into a humidified anaerobic chamber containing $94 \% \mathrm{~N}_{2}, 5 \% \mathrm{CO}_{2}$, and $1 \% \mathrm{O}_{2}$ for $48 \mathrm{~h}$. Propofol (Sigma-Aldrich, St. Louis, MO, USA) was primarily dissolved in dimethyl sulfoxide (DMSO) shortly before use, and it was diluted into RPMI 1640 medium to yield a final propofol concentration of $10 \mu \mathrm{g} / \mathrm{mL}$. Propofol $(10 \mu \mathrm{g} / \mathrm{mL})$ was applied to stimulate PC-12 cells for $48 \mathrm{~h}$. The final concentration of DMSO in the control group and propofol group was $0.1 \%$. The culture medium was refreshed every other day.

\section{Transient transfection with miRNAs}

Scramble miRNAs, miR-153 mimic, miR-153 inhibitor, and negative control of miR-153 inhibitor (NC) were purchased from GenePharma Co. (Shanghai, China). Each miRNA was transfected into PC-12 cells using Lipofectamine 3000 reagent (Invitrogen, Carlsbad, CA, USA) following the manufacturer's protocol.

\section{Generation of stably transfected cells}

Short-hairpin RNA directed against BTG3 or a non-targeting sequence was sub-cloned into pGPU6 plasmid (GenePharma), and the recombined plasmids were referred to as sh-BTG3 and sh-NC, respectively. Full-length BTG3 sequence was amplified and inserted into pcDNA3.1 plasmid (GenePharma) to construct pc-BTG3. Recombined plasmids as well as pcDNA3.1 were transfected into PC-12 cells using the lipofectamine 3000 reagent (Invitrogen) according to the manufacturer's instructions. Stable transfection was selected after incubation in culture medium containing $0.5 \mathrm{mg} / \mathrm{ml}$ G418 (Sigma-Aldrich) for approximately 4 weeks.

\section{Cell viability assay}

Cells, seeded in 96-well plates at a density of $5 \times 10^{3}$ cells/well, were incubated at $37^{\circ} \mathrm{C}$ overnight. Then, after treatments, $10 \mu \mathrm{L}$ of Cell Counting Kit-8 (CCK-8) solution, provided by the Dojindo Molecular Technologies (Gaithersburg, MD, USA) was added into the culture medium. Cells were incubated at $37^{\circ} \mathrm{C}$ for additional $1 \mathrm{~h}$, and the absorbance at $450 \mathrm{~nm}$ was measured using a Microplate Reader (Bio-Rad, Hercules, CA, USA).

\section{Apoptosis assay}

After treatments, cells were trypsinized $(0.25 \%$ trypsinEDTA) and collected. Then, cells were washed in cold phosphate-buffered saline (PBS), resuspended in binding buffer provide by the FITC Annexin V/Dead Cell Apoptosis Kit (Invitrogen), and were stained with FITC Annexin V and propidium iodide (PI) according to the instructions of the kit. Stained cells were identified and quantified using a FACS can (Beckman Coulter, Fullerton, CA, USA). Data were analyzed by using FlowJo software (Tree Star, San Carlos, CA, USA).

miRNA detection by stem-loop reverse transcription (RT)PCR

After treatments, total RNA was extracted from treated cells using the RNAeasy Mini Kit (Qiagen Sciences, Germantown, MD, USA). cDNAs specific to miRNAs were synthesized using the Taqman MicroRNA Reverse Transcription Kit (Applied Biosystems, Foster City, CA, USA) 
in line with the suggested procedure. The thermocycling program was shown as follows: $30 \mathrm{~min}$ at $16^{\circ} \mathrm{C}, 30 \mathrm{~min}$ at $42^{\circ} \mathrm{C}$ and $5 \mathrm{~min}$ at $85^{\circ} \mathrm{C}$. Afterwards, real-time PCR was performed with the help of the Taqman Universal Master Mix II (Applied Biosystems) according to the manufacturer's protocol, and cDNA (50 ng) was acted as the template. The thermal cycling conditions were 1 cycle at $95^{\circ} \mathrm{C}$ for $10 \mathrm{~min}$, followed by 40 cycles of $95^{\circ} \mathrm{C}$ for $15 \mathrm{~s}$ and $60^{\circ} \mathrm{C}$ for $1 \mathrm{~min}$. Relative expression of miR-153 was calculated on the basis of the $2^{-\Delta \Delta C t}$ method [24], and U6 was acted as the housekeeping gene.

\section{Western blot analysis}

After treatments, treated cells were suspended in RIPA buffer (Beyotime, Shanghai, China), and the proteins in the supernatants of the whole lysates were quantified using the BCA Assay Kit (Beyotime). Protein samples ( $40 \mu \mathrm{g} /$ lane) were separated by SDS-PAGE, followed by blotting onto the polyvinylidene difluoride (PVDF) membranes. After blocking in 5\% non-fat milk at room temperature for $1 \mathrm{~h}$, membranes were incubated with primary antibodies and HRP-conjugated secondary antibodies (goat anti-rabbit, ab97051; goat anti-mouse, ab205719; both Abcam, Cambridge, UK), successively. Primary antibodies included antibodies against cleaved caspase-3 (ab49822), pro caspase-3 (ab90437), B cell lymphoma-2 (Bcl-2; ab196495), Bcl-2-associated X protein (Bax; ab182733), mechanistic target of rapamycin (mTOR, ab2732), phospho (p)-mTOR (ab137133), $\beta$-actin (ab8229, all Abcam), B-cell translocation gene 3 (BTG3; TA323849, OriGene Technologies, Inc., Rockville, MD, USA), caspase-9 (\#9508), p70S6K (\#9202), p-p70S6K (\#9205), AMP-activated protein kinase (AMPK; \#5832) or p-AMPK (\#50081, all Cell Signaling Technology, Beverly, MA, USA). Membranes were then washed by Tris-buffered saline containing $0.1 \%$ Tween-20 (TBST). Proteins in the PVDF membranes were visualized by using an enhanced chemiluminescence kit (GE Healthcare Bio-Sciences, Pittsburgh, PA, USA). The intensity of the bands was determined by Image J software (National Institutes of Health, Bethesda, MA, USA).

\section{Statistical analysis}

Experiments were performed in triplicate with three repeats. The results were presented as the mean \pm standard deviation (SD). Statistical analysis was performed using Graphpad Prism 5 software (GraphPad, San Diego, CA, USA). The $P$-values were calculated using unpaired two-tailed $t$-test, one-way or two-way analysis of variance (ANOVA). A $P<0.05$ was considered as a significant difference.

\section{Results}

Hypoxia induced PC-12 cell apoptosis

After hypoxia treatments, cell viability and apoptosis of PC-12 cells were evaluated. Compared with cells under normoxia, cell viability was markedly reduced by hypoxia exposure $(P<0.01$, Fig. 1a). Percentage of apoptotic cells in the Hypoxia group was notably higher than that in the Normoxia group $(P<0.01$, Fig. $1 \mathrm{~b})$. Western blot results in Fig. 1c showed expression of pro-apoptotic cleaved caspase-3, cleaved caspase- 9 and Bax was observably up-regulated after hypoxia exposure, whereas expression of anti-apoptotic Bcl-2 was down-regulated after hypoxia exposure. Results proposed that in vitro hypoxia model was constructed successfully in PC-12 cells.

\section{Propofol alleviated hypoxia-induced apoptosis in PC-12 cells}

PC-12 cells under normoxia were treated with propofol, and cell viability and apoptosis were measured. As shown in Fig. 2a, difference of viability between propofol-treated cells and untreated cells was non-significant $(P>0.05)$. Propofol also showed a non-significant effect on apoptosis in cells under normoxia $(P>0.05$, Fig. $2 b)$. After that, cells were exposed to hypoxia with or without propofol, and then cell viability and apoptosis were measured. Compared with the Hypoxia group, cell viability was markedly enhanced $(P<0.05$, Fig. 2c) and apoptosis was notably
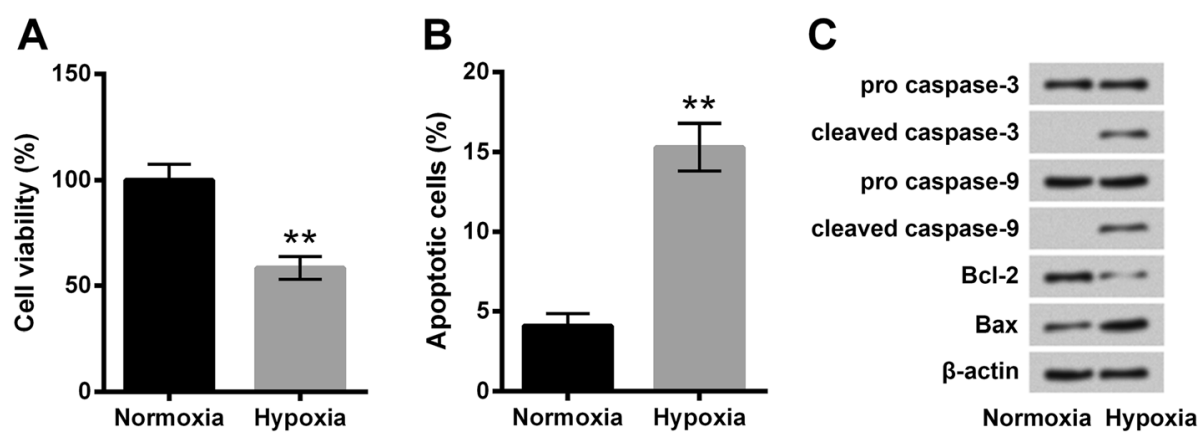

Fig. 1 Hypoxia induced PC-12 cell apoptosis. a Cell viability by CCK-8. b Percentage of apoptotic cells by flow cytometry assay. c Expression of apoptosis-related proteins by Western blot analysis. PC-12 cells were incubated under normoxia or hypoxia for $48 \mathrm{~h}$. Data was presented as the mean \pm standard deviation (SD) of three independent experiments. ${ }^{* *}, P<0.01$ 


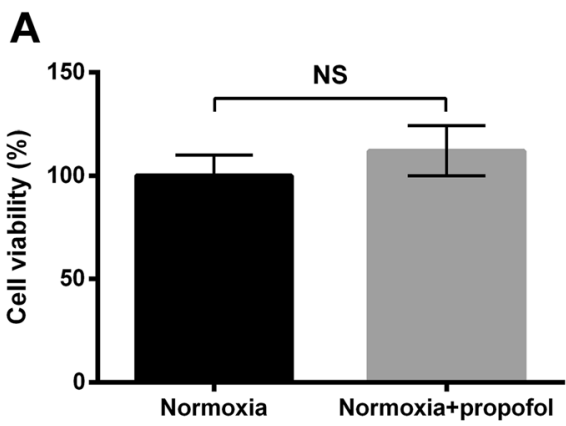

C

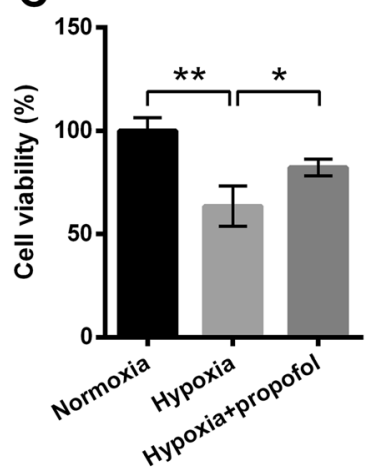

B

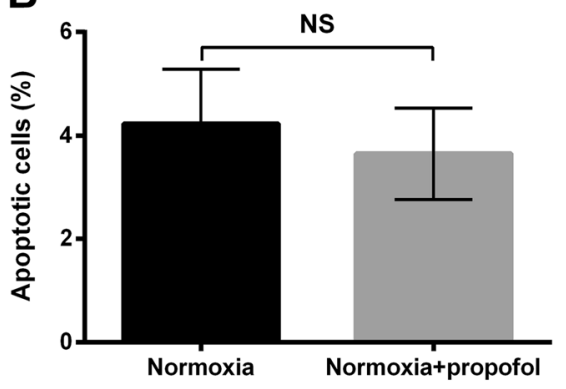

E

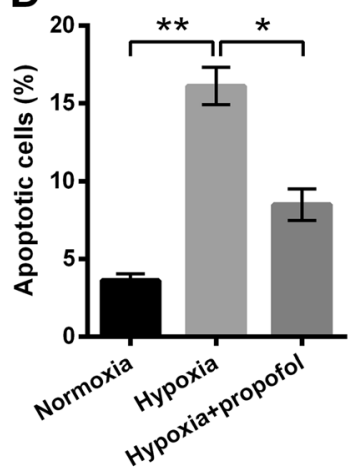

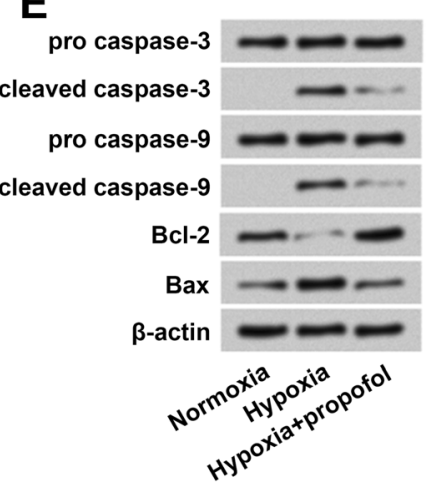

Fig. 2 Hypoxia-induced PC-12 cell apoptosis was alleviated by propofol. a Cell viability by CCK-8. b Percentage of apoptotic cells by flow cytometry assay. c Cell viability by CCK-8. d Percentage of apoptotic cells by flow cytometry assay. e Expression of apoptosis-related proteins by Western blot analysis. PC-12 cells were incubated under normoxia or hypoxia with or without $10 \mu \mathrm{g} / \mathrm{mL}$ propofol for $48 \mathrm{~h}$. Data was presented as the mean \pm standard deviation (SD) of three independent experiments. NS, $P>0.05 ;{ }^{*}, P<0.05 ;{ }^{* *}, P<0.01$

decreased $(P<0.05$, Fig. $2 \mathrm{~d})$ by propofol. In the meantime, alteration of apoptosis-related proteins was also attenuated by propofol (Fig. 2e). Therefore, we concluded that propofol could attenuate hypoxia-induced apoptosis in PC-12 cells.

\section{Propofol up-regulated miR-153 expression in PC-12 cells} Cells under normoxia were stimulated with propofol, and results in Fig. 3a showed propofol dramatically up-regulated
miR-153 expression compared with the Normoxia group $(P<0.001)$. After exposure to hypoxia with or without propofol, the expression of miR-153 in PC-12 cells was also determined. As evidenced in Fig. 3b, we found miR-153 level was significantly elevated by hypoxia treatments $(P<0.01)$, and the elevation was further increased by propofol $(P<0.05)$. Results suggested that miR-153 might be involved in the regulatory effects of propofol on hypoxia-treated PC-12 cells.
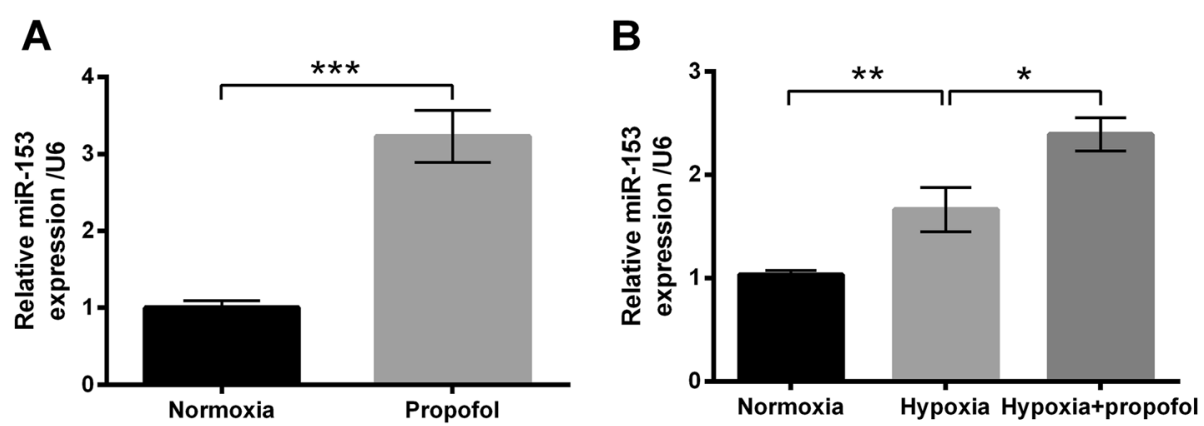

Fig. 3 Propofol up-regulated miR-153 expression in PC-12 cells. a Expression of miR-153 by stem-loop RT-PCR. Cells were incubated under hypoxia with or without propofol for $48 \mathrm{~h}$. Cells in the Normoxia group were incubated under normoxia for $48 \mathrm{~h}$. PC-12 cells were incubated under normoxia with or without $10 \mu \mathrm{g} / \mathrm{mL}$ propofol for $48 \mathrm{~h}$. b Expression of miR-153 by stem-loop RT-PCR. PC-12 cells were incubated under normoxia or hypoxia with or without $10 \mu \mathrm{g} / \mathrm{mL}$ propofol for $48 \mathrm{~h}$. Data was presented as the mean \pm standard deviation (SD) of three independent experiments. ${ }^{*}, P<0.05 ;{ }^{*}, P<0.01 ;{ }^{* *}, P<0.001$ 
Propofol protected PC-12 cells against hypoxia through up-regulating $\mathrm{miR}-153$

After transfection with miRNAs, miR-153 level in cells transfected with miR-153 inhibitor was markedly lower than that in cells transfected with $\mathrm{NC}(P<0.01$, Fig. 4a), indicating that cell transfection could down-regulate miR-153 expression successfully. Then, transfected or untransfected cells were exposed to hypoxia with or without propofol, and then cell viability and apoptosis were analyzed. Results showed effects of propofol on PC-12 cells under hypoxia could be significantly reversed by miR-153 inhibition, as evidenced by decreased cell viability $(P<0.01$, Fig. $4 b)$, increased apoptotic cells $(P<0.05$, Fig. 4c), up-regulation of cleaved caspase-3, cleaved caspase-9 and Bax, and down-regulation of Bcl-2 (Fig. 4d). Moreover, the effects of miR-153 on hypoxia-treated cells were explored to verify whether alteration of miR-153 could effects hypoxia-treated cells. Results showed effects of hypoxia were augmented by miR-153 inhibition, as miR-153 inhibition significantly decreased cell viability
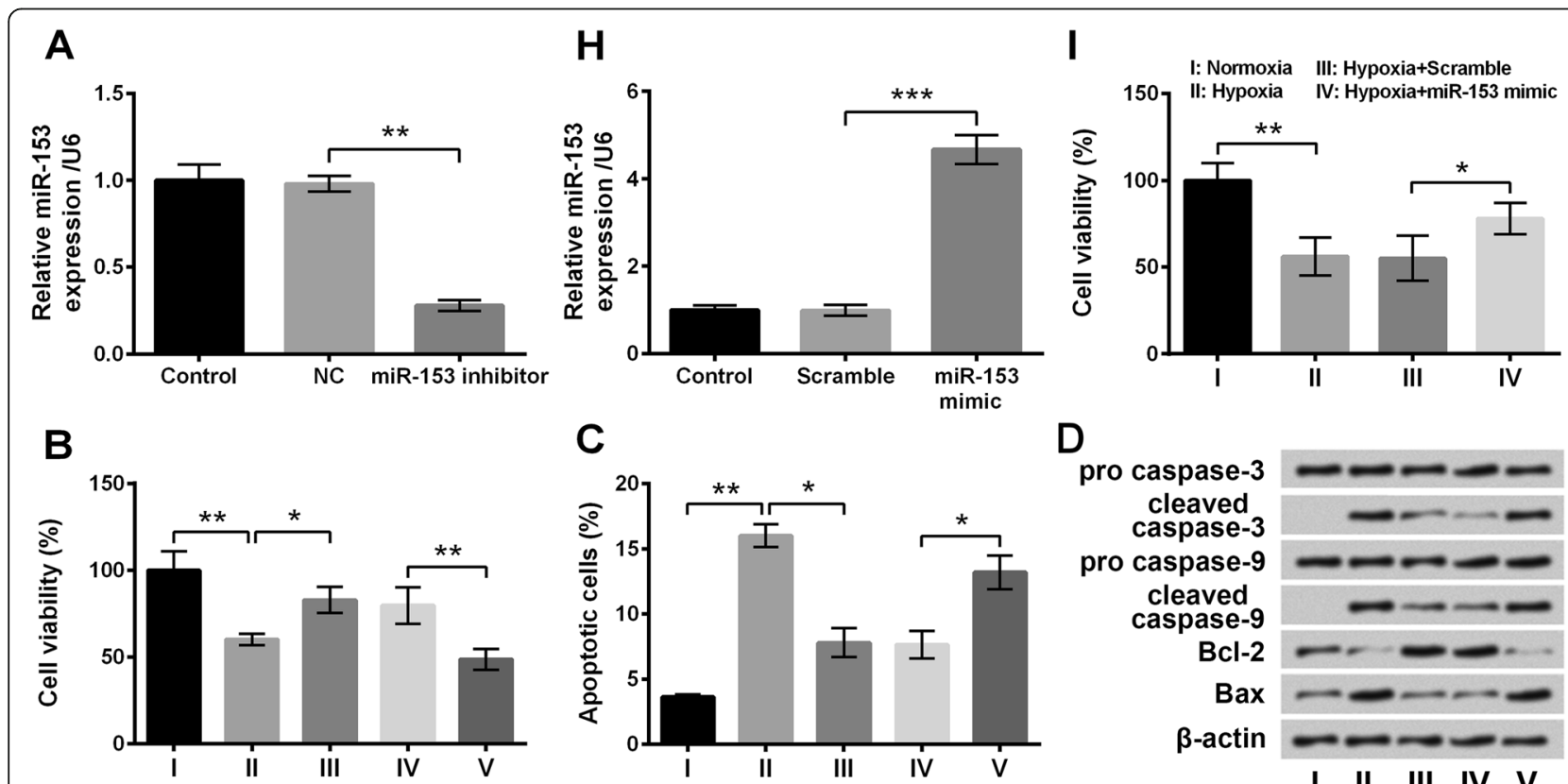

C
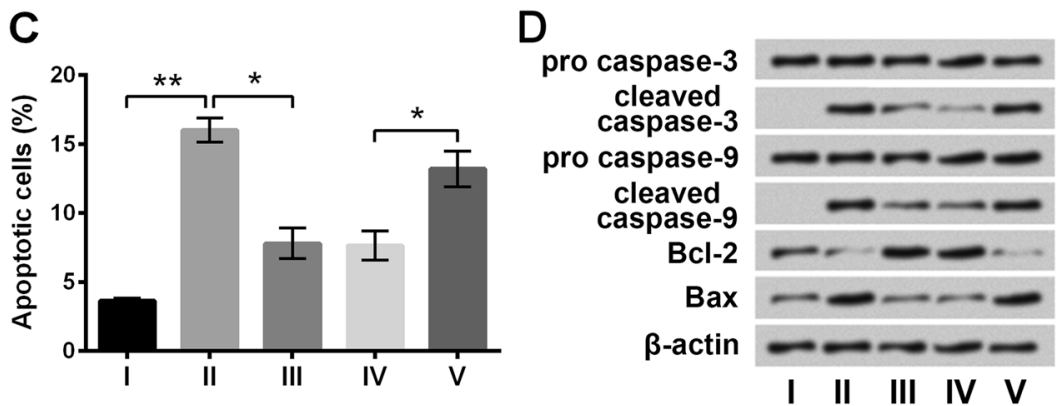

I: Normoxia II: Hypoxia III: Hypoxia+propofol IV: Hypoxia+propofol+NC

\section{V: Hypoxia+propofol miR-153 inhibitor}

E

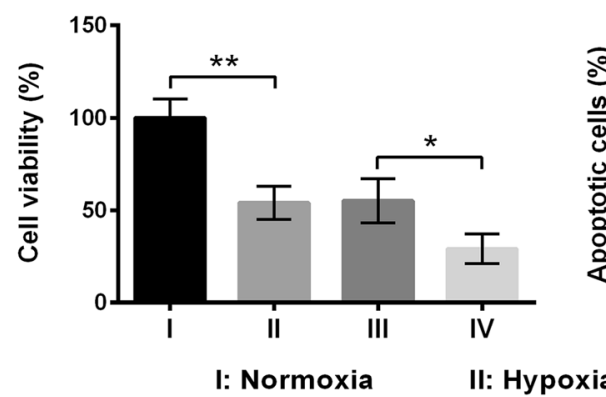

$\mathbf{F}$

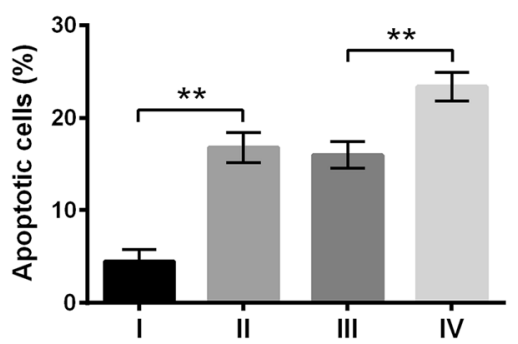

G

pro caspase-3

cleaved caspase-3

pro caspase-9

cleaved caspase-9

BCl-2

Bax

$\beta$-actin

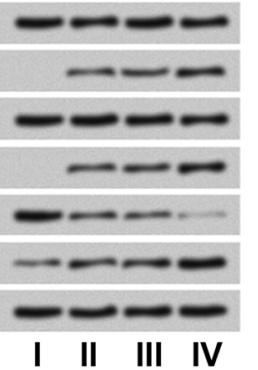

Fig. 4 Effects of propofol on hypoxia-injured PC-12 cells were reversed by miR-153 inhibition. a Expression of miR-153 by stem-loop RT-PCR. Cells were divided into five groups, including Normoxia, Hypoxia, Hypoxia + propofol, Hypoxia + propofol + NC, and Hypoxia + propofol + miR-153 inhibitor. The duration of treatments was $48 \mathrm{~h}$. b Cell viability by CCK-8. c Percentage of apoptotic cells by flow cytometry assay. $\mathbf{d}$ Expression of apoptosis-related proteins by Western blot analysis. Cells were divided into four groups, including Normoxia, Hypoxia, Hypoxia + NC, and Hypoxia + miR-153 inhibitor. The duration of treatments was $48 \mathrm{~h}$. e Cell viability by CCK-8. f Percentage of apoptotic cells by flow cytometry assay. $\mathbf{g}$ Expression of apoptosis-related proteins by Western blot analysis. Cells were transfected with scramble miRNAs or miR-153 mimic. $\mathbf{h}$ Expression of miR-153 by stem-loop RT-PCR. i Cell viability by CCK-8 assay. Cells were divided into four groups, including Normoxia, Hypoxia, Hypoxia + Scramble, and Hypoxia + miR-153 mimic. The duration of treatments was $48 \mathrm{~h}$. Data was presented as the mean \pm standard deviation (SD) of three independent experiments. ${ }^{*}, P<0.05 ;{ }^{* *}, P<0.01 ;{ }^{* * *}, P<0.001$ 
$(P<0.05$, Fig. 4e $)$ while markedly increased apoptotic cells $(P<0.01$, Fig. $4 \mathrm{f})$, as compared to the Hypoxia + NC group. As shown in Fig. 4h, miR-153 level in cells transfected with miR-153 mimic was remarkably higher than that in cells transfected with Scramble miRNA $(P<0.001)$, indicating that cell transfection could up-regulate miR-153 expression successfully. Results in Fig. 4i showed miR-153 overexpression could effectively enhanced cell viability compared with the Hypoxia + Scramble group $(P<0.05)$. Results collectively suggested that propofol might affect hypoxia-injured PC-12 cells through up-regulating miR-153 expression.

\section{BTG3 expression was negatively correlated with miR-153 expression}

The protein expression of BTG3 in PC-12 cells abnormally expressing miR-153 was assessed. In Fig. 5a, BTG3 protein expression level in cells overexpressing miR-153 was significantly lower than that in the Scramble group $(P<0.05)$. In the meantime, BTG3 protein expression level in cells silencing miR-153 was significantly higher than that in the NC group $(P<0.05$, Fig. 5b). Results indicated that BTG3 expression was negatively correlated with miR-153 expression in PC-12 cells.

BTG3 overexpression inactivated the mTOR pathway and AMPK activation in PC-12 cells

The effects of BTG3 on activation of the mTOR pathway and AMPK were analyzed. In stably transfected cells, protein expression of BTG3 was testified. As shown in Fig. 6a, BTG3 protein expression level in cells transfected with pc-BTG3 was dramatically higher than that in cells transfected with pcDNA3.1 $(P<0.01)$. Meanwhile, BTG3 protein expression level in cells transfected with sh-BTG3 was significantly lower than that in cells transfected with sh-NC $(P<0.01)$. Results proved that BTG3 expression could be overexpressed or silenced after stable transfection. Then, phosphorylation of AMPK and key kinases in the mTOR pathway was analyzed. Phosphorylated levels of mTOR, p70S6K and AMPK were markedly reduced by BTG3 overexpression $(P<0.05$ or $P<0.01$, Fig. $6 \mathrm{~b}, \mathrm{c})$ while were dramatically enhanced by BTG3 silence $(P<0.01$ or $P<0.001)$. Results indicated that BTG3 overexpression could inhibit the mTOR pathway and AMPK activation in PC-12 cells.

\section{Hypoxia inactivated the mTOR pathway and AMPK activation, and propofol attenuated these inhibitory effects via regulation of BTG3}

The effects of hypoxia and/or propofol on BTG3 expression were evaluated. As shown in Fig. 7a, BTG3 expression was up-regulated by hypoxia while was down-regulated by propofol, and the effects of hypoxia could be alleviated by propofol. Moreover, we also found the phosphorylated levels of mTOR, p70S6K and AMPK were reduced by hypoxia while were enhanced by propofol (Fig. 7b, c). In addition, the effects of hypoxia on the mTOR pathway and AMPK activation were attenuated by propofol. Results indicated that hypoxia inactivated the mTOR pathway and AMPK activation, and propofol attenuated these inhibitory effects via regulation of BTG3.

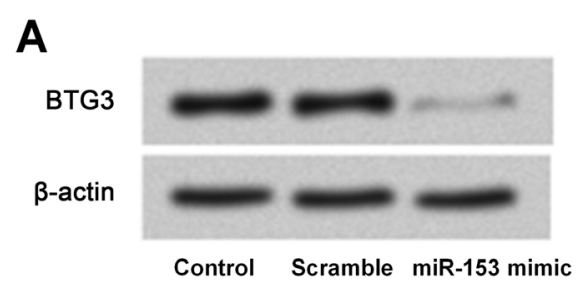

B
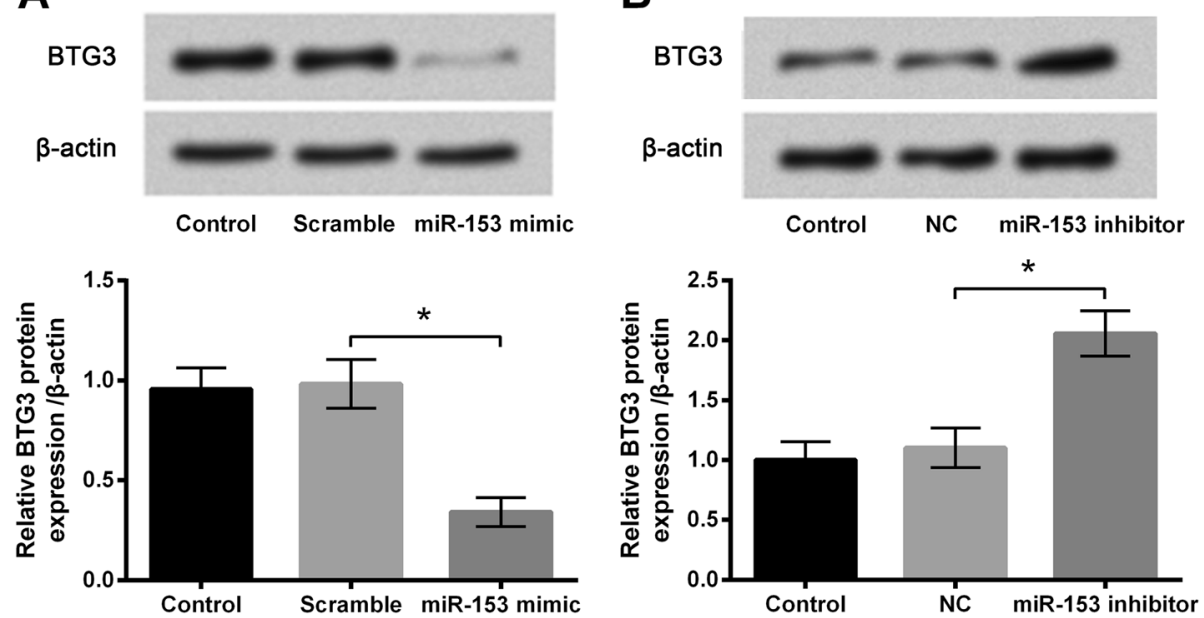

Fig. 5 BTG3 was negatively regulated by miR-153 expression in PC-12 cells. a PC-12 cells were transfected with scramble miRNAs or miR-153 mimic. b PC-12 cells were transfected with miR-153 inhibitor or its negative control (NC). Protein expression of BTG3 was measured by Western blot analysis. Untransfected cells were acted as control Data was presented as the mean \pm standard deviation (SD) of three independent experiments. ${ }^{*}, P<0.05$ 

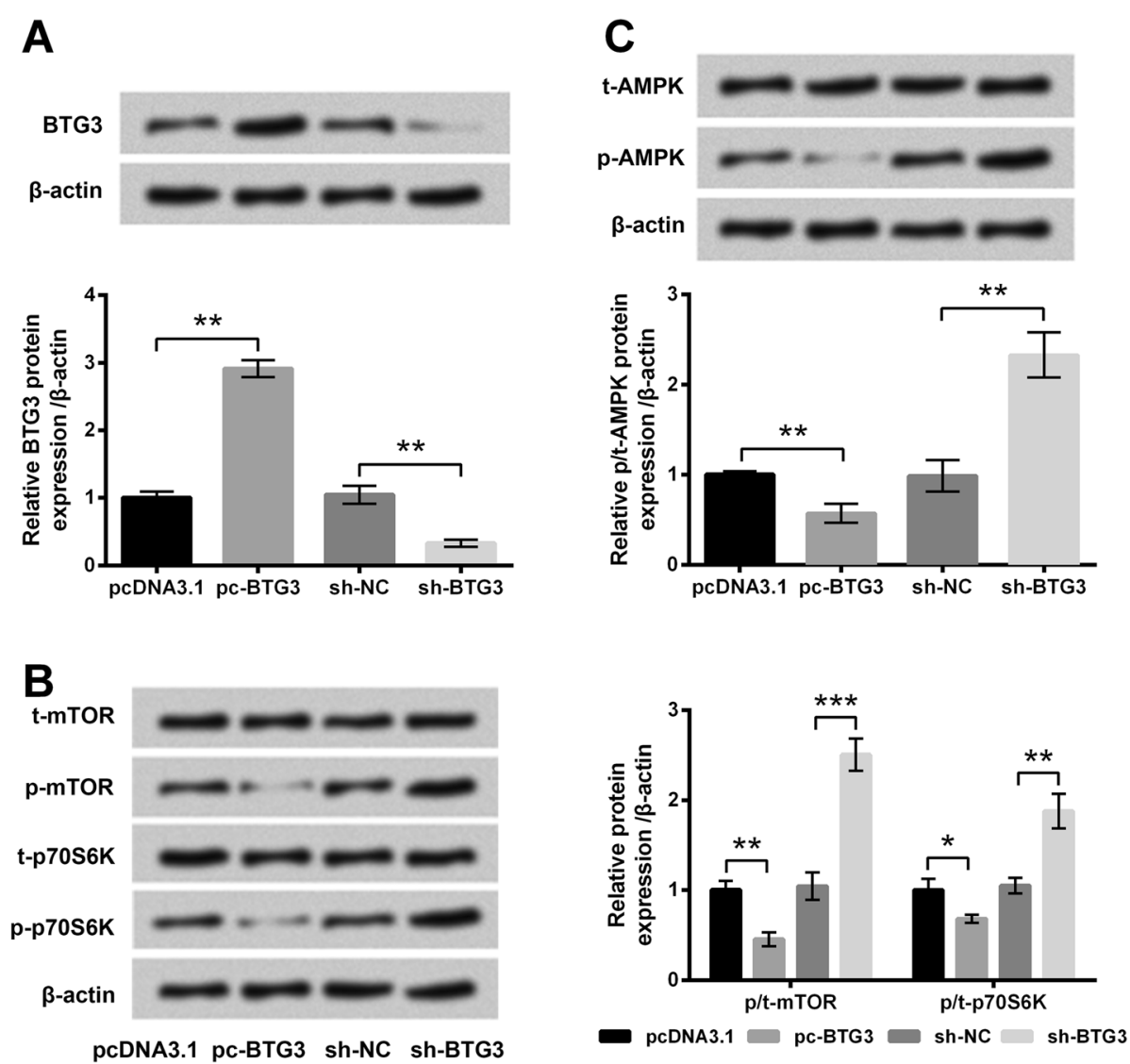

Fig. 6 BTG3 overexpression inhibited the mTOR pathway and AMPK activation in PC-12 cells. a Protein expression of BTG3 was assessed by Western blot analysis. Phosphorylated levels of key kinases in the mTOR pathway (b) and AMPK (c) were assessed by Western blot analysis. PC-12 cells were transfected with pcDNA3.1, pc-BTG3, sh-NC or sh-BTG3. Data was presented as the mean \pm standard deviation (SD) of three independent experiments. ${ }^{*}, P<0.05{ }^{*}{ }^{*}, P<0.01 ;{ }^{* *}, P<0.001$
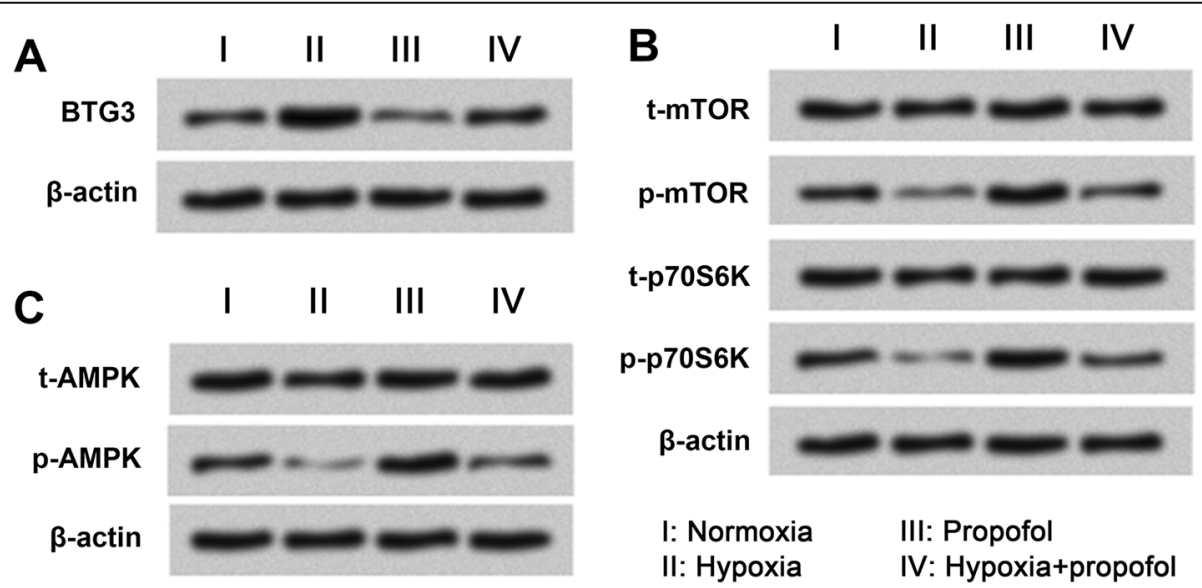

\section{I: Normoxia III: Propofol \\ II: Hypoxia IV: Hypoxia+propofol}

Fig. 7 Hypoixa inhibited the mTOR pathway and AMPK activation, and propofol attenuated these inhibitory effects via regulation of BTG3. PC-12 cells were divided into four groups, including Normoxia, Hypoxia, Propofol, and Hypoxia + propofol. a Protein expression of BTG3 by Western blot analysis. Phosphorylated levels of key kinases in the mTOR pathway (b) and AMPK (c) were assessed by Western blot analysis 


\section{Discussion}

PC-12 cells are widely used as a model system to study the mechanisms involved in neuronal cells under hypoxia. For example, the protective role of silent information regulation 2 homolog 3 (SIRT3) in ischemia-induced neuronal death as well as the underlying mechanisms was studied in PC-12 cells [25]. The neuroprotective role of cerebrolysin in hypoxia-induced neuronal cytotoxicity was also studied in PC-12 cells [26]. In the present study, the in vitro protective role of propofol in neuronal cells was studied in PC-12 cells. Data indicated that hypoxia-induced PC-12 cell injury could be alleviated by propofol treatment. Then, we found miR-153 level was elevated after hypoxia treatment and its expression was further up-regulated in cells treated with hypoxia and propofol. Additional experiments revealed that propofol functioned through up-regulating miR-153 in hypoxia-exposed cells. Afterwards, we found miR-153 expression negatively regulated BTG3 expression, and BTG3 inhibited the mTOR pathway and AMPK activation in PC-12 cells.

In the first step of our study, the effects of propofol on hypoxia-treated cells were measured. Although propofol showed non-significant effects on viability and apoptosis in cells under normoxia, the hypoxia-induced decrease of cell viability as well as increase of apoptosis was mitigated by propofol in PC-12 cells. Apoptosis is a process of programmed cell death, in which several changes were observed, such as alteration of Bcl-2 family proteins, activation of caspases and DNA fragmentation [27]. In our study, hypoxia-induced up-regulation of Bax, cleaved caspase- 3 and cleaved caspase- 9 as well as down-regulation of Bcl-2 was obviously attenuated by propofol, implying that propofol might alleviate hypoxia-induced PC-12 cell injury through repressing the mitochondrial- and caspase-dependent pathways. The protective role of propofol against hypoxia injury in $\mathrm{PC}-12$ cells was consistent with that in other cell types. For example, cell death induced by hypoxia in alveolar epithelial type II cells of rats could be reduced by propofol [10]. hypoxia/reoxygenation-induced apoptosis of human brain vascular smooth muscle cells was attenuated by propofol [11].

Next, we explored whether there was a correlation between propofol and miR-153 in hypoxia-injured PC-12 cells. Intriguingly, our results found miR-153 level was up-regulated by hypoxia, and its level was further up-regulated by propofol in PC-12 cells under normoxia or hypoxia. An evidence suggested that the increased expression of miR-153 occurred in cells in response to external stimulus, for instance, neurotoxin [23]. Similarly, although it's still unexplored why hypoxia could up-regulate the expression of miR-125, we considered that the expression of miR-125 was enhanced against hypoxia-induced injury. However, the increased miR-125 could not thoroughly repress the expression of hypoxiainducible factor (HIF)-1 $\alpha$, which was inhibited by the overexpression of miR-153 for inhibiting apoptosis and enhancing proliferation [28, 29]. By adequately enhancing the expression of miR-153, propofol exerted a cytoprotection against hypoxia-induced injury. Moreover, neuroprotective effects of propofol on hypoxia-injured PC-12 cells could be abrogated by miR-153 knockdown, indicating that up-regulated miR-153 might be a possible reason for the neuroprotective role of propofol. To support the conclusion, we also proved that hypoxia injury was heightened by miR-153 inhibition while was attenuated by miR-153 overexpression. A previous study has proposed the negative regulation between miR-153 and hypoxia-inducible factor (HIF)-1 $\alpha$ [29]. However, the effects of HIF- $1 \alpha$ on hypoxia-injury are controversial. Liu et al. have shown that down-regulation of HIF-1 $\alpha$ increases apoptosis of hypoxic cardiomyocytes [30]. Chen et al. have reported that HIF-1 $\alpha$ knockdown alleviates neonatal brain damage after hypoxic injury. Therefore, there might be another downstream factor of miR-153 in the regulation of propofol.

BTG3 belonging to anti-proliferative BTG gene family has been reported as a tumor suppressor gene [31]. A previous study has illustrated that BTG3 overexpression showed higher expression of Bax, caspase- 3 and caspase- 9 [32]. Considering the observable effects of propofol on those proteins associated with apoptosis, we speculated that BTG3 might participate in the regulatory mechanism of propofol. Results in our study found BTG3 expression was negatively regulated by miR-153. A previous study has proved that BTG3 is a direct target of p53 [33]. Krüppel-like factor 5 (KLF5) is a target of miR-153 [34] which can interact with p53 [35]. Those observations described above might provide a rational explanation for the negative correlation between miR-153 and BTG3. The mTOR pathway governing cellular response to hypoxia plays critical role in regulating cell death under environmental stress [36]. AMPK is a stress-responsive enzyme involved in cell adaptation to an energy crisis [37]. We further found that BTG3 overexpression could inhibited the mTOR pathway and AMPK activation, and BTG3 silence showed the opposite effects.

In the end, we also studied the effects of hypoxia and/ or propofol on BTG3 expression and phosphorylation of mTOR, p70S6K and AMPK, in order to verify the regulatory axis of propofol-miR-153-BTG3. Western blot results showed hypoxia up-regulated BTG3 expression while propofol down-regulated BTG3 expression, and the hypoxia-induced BTG3 abundance was decreased by propofol. The effects of hypoxia and/or propofol on the mTOR pathway and AMPK activation could substantiate the effects of BTG3 on the mTOR pathway and AMPK activation. 


\section{Conclusions}

To summarize, we verified the protective role of propofol in hypoxia-exposed PC-12 cells and found propofol might affect PC-12 cells under hypoxia through miR-153-mediated down-regulation of BTG3. BTG3 expression overexpression inhibited the mTOR pathway and AMPK activation. This study provided basis for the study of propofol function, assisting in discovery of innovative strategies for clinical neuroprotection.

\section{Acknowledgements}

None.

\section{Funding}

The work was supported by grants from the Beijing Municipal Administration of Hospitals Clinical Medicine Development of Special Funding Support (ZYLX201810).

\section{Availability of data and materials}

Data sharing not applicable to this article as no datasets were generated or analysed during the current study.

\section{Authors' contributions}

JM conceived the study; HS, XG and JP carried out the experiments; YH, XW and $\mathrm{YL}$ conducted the analyses; and $\mathrm{MH}$ wrote the paper. All authors have read and approved the manuscript, and ensure that this is the case.

\section{Ethics approval and consent to participate}

Not applicable.

\section{Consent for publication}

Not applicable.

\section{Competing interests}

The authors declare that they have no competing interests.

\section{Publisher's Note}

Springer Nature remains neutral with regard to jurisdictional claims in published maps and institutional affiliations.

Received: 18 April 2018 Accepted: 3 December 2018

Published online: 22 December 2018

\section{References}

1. Georgia MAD. Brain tissue oxygen monitoring in Neurocritical care. J Intensive Care Med. 2015;30(8):473-83.

2. Yang Z-B, Luo X-J, Ren K-D, Peng J-J, Tan B, Liu B, Lou Z, Xiong X-M, Zhang $X-J$, Ren $X$, et al. Beneficial effect of magnesium lithospermate $B$ on cerebral ischemia-reperfusion injury in rats involves the regulation of miR-107/ glutamate transporter 1 pathway. Eur J Pharmacol. 2015:766:91-8.

3. Sakamoto M, Miyazaki Y, Kitajo K, Yamaguchi A. VGF, which is induced transcriptionally in stroke brain, enhances neurite extension and confers protection against ischemia in vitro. Transl Stroke Res. 2015;6(4):301-8.

4. Roger VL, Go AS, Lloyd-Jones DM, Adams RJ, Berry JD, Brown TM, Carnethon MR, Dai S, de Simone G, Ford ES et al: Heart disease and stroke statistics--2011 update: a report from the American Heart Association. Circulation 2011, 123(4):e18-e209.

5. Yang SH, Lou M, Luo B, Jiang WJ, Liu R. Precision medicine for ischemic stroke, let us move beyond time is brain. Transl Stroke Res. 2018;9(2):93-5.

6. Xiong M, Shiwalkar N, Reddy K, Shin P, Bekker A. Neurobiology of Propofol addiction and supportive evidence: what is the new development? Brain Sciences. 2018;8(2):36.

7. Gelegen C, Miracca G, Ran MZ, Harding EC, Ye Z, Yu X, Tossell K, Houston CM, Yustos R, Hawkins ED, et al. Excitatory pathways from the lateral Habenula enable Propofol-induced sedation. Curr Biol. 2018;28(4): 580-587.e585.
8. Zhang D, Zhou XH, Zhang J, Zhou YX, Ying J, Wu GQ, Qian JH. Propofol promotes cell apoptosis via inhibiting HOTAIR mediated mTOR pathway in cervical cancer. Biochem Biophys Res Commun. 2015;468(4):561-7.

9. Zhang L, Wang N, Zhou S, Ye W, Jing G, Zhang M. Propofol induces proliferation and invasion of gallbladder cancer cells through activation of Nrf2. J Exp Clin Cancer Res. 2012;31:66.

10. Ning HJ, Yuan HB, Xu HT, He XY. Propofol reduces hypoxiainduced autophagic cell death through downregulating HIF 1alpha in alveolar epithelial type II cells of rats. Mol Med Rep. 2017;16(2):1509-15.

11. Zhang J, Xia Y, Xu Z, Deng X. Propofol suppressed hypoxia/Reoxygenationinduced apoptosis in HBVSMC by regulation of the expression of $\mathrm{BCl}-2$, Bax, Caspase3, Kir6.1, and p-JNK. Oxidative Med Cell Longev. 2016;2016:1518738.

12. Zhang DX, Ding HZ, Jiang S, Zeng YM, Tang QF. An in vitro study of the neuroprotective effect of propofol on hypoxic hippocampal slice. Brain Inj. 2014:28(13-14):1758-65.

13. Lu Y, Chen W, Lin C, Wang J, Zhu M, Chen J, Miao C. The protective effects of propofol against $\mathrm{CoCl}$-induced $\mathrm{HT} 22$ cell hypoxia injury via PP2A/ CAMKIlalpha/nNOS pathway. BMC Anesthesiol. 2017;17(1):32.

14. Ulbrich F, Eisert L, Buerkle H, Goebel U, Schallner N. Propofol, but not ketamine or midazolam, exerts neuroprotection after ischaemic injury by inhibition of toll-like receptor 4 and nuclear factor kappa-light-chainenhancer of activated B-cell signalling: a combined in vitro and animal study. Eur J Anaesthesiol. 2016;33(9):670-80.

15. Kaushik SB, Kaushik N. Non-coding RNAs in skin cancers: an update. Noncoding RNA Research. 2016;1(1):83-6.

16. Luan $Y$, Zhang $X$, Zhang Y, Dong Y. MicroRNA-210 protects $P C-12$ cells against hypoxia-induced injury by targeting BNIP3. Front Cell Neurosci. 2017;11:285

17. Han L, Dong Z, Liu N, Xie F, Wang N. Maternally expressed gene 3 (MEG3) enhances PC12 cell hypoxia injury by targeting MiR-147. Cell Physiol Biochem. 2017:43(6):2457-69.

18. Hao W, Zhao ZH, Meng QT, Tie ME, Lei SQ, Xia ZY. Propofol protects against hepatic ischemia/reperfusion injury via miR-133a-5p regulating the expression of MAPK6. Cell Biol Int. 2017:41(5):495-504.

19. Wu Z, He B, He J, Mao X. Upregulation of miR-153 promotes cell proliferation via downregulation of the PTEN tumor suppressor gene in human prostate cancer. Prostate. 2013;73(6):596-604

20. Shan N, Shen L, Wang J, He D, Duan C. MiR-153 inhibits migration and invasion of human non-small-cell lung cancer by targeting ADAM19. Biochem Biophys Res Commun. 2015:456(1):385-91.

21. Liang H, Xiao J, Zhou Z, Wu J, Ge F, Li Z, Zhang H, Sun J, Li F, Liu R, et al. Hypoxia induces miR-153 through the IRE1alpha-XBP1 pathway to fine tune the HIF1alpha/NEGFA axis in breast cancer angiogenesis. Oncogene. 2018.

22. Kim JH, Kim BK, Kim DW, Shin HY, Yu SB, Kim DS, Ryu SJ, Kim KH, Jang HK, Kim JD. Effect of Propofol on microRNA expression profile in adipocytederived adult stem cells. Chonnam Med J. 2014;50(3):86-90.

23. Fragkouli A, Doxakis E. miR-7 and miR-153 protect neurons against $\mathrm{MPP}(+)$-induced cell death via upregulation of mTOR pathway. Front Cell Neurosci. 2014:8:182

24. Livak KJ, Schmittgen TD. Analysis of relative gene expression data using real-time quantitative PCR and the 2(-Delta Delta $C(T))$ method. Methods. 2001;25(4):402-8.

25. Wang Q, Li L, Li CY, Pei Z, Zhou M, Li N. SIRT3 protects cells from hypoxia via PGC-1alpha- and MnSOD-dependent pathways. Neuroscience. 2015;286:109-21.

26. Hartwig $\mathrm{K}$, Fackler $\mathrm{V}$, Jaksch-Bogensperger $\mathrm{H}$, Winter $\mathrm{S}$, Furtner $\mathrm{T}$, CouillardDespres S, Meier D, Moessler H, Aigner L. Cerebrolysin protects PC12 cells from CoCl2-induced hypoxia employing GSK3beta signaling. Int J Dev Neurosci. 2014;38:52-8

27. Chio CC, Wei L, Chen TG, Lin CM, Shieh JP, Yeh PS, Chen RM. Neuron-derived orphan receptor 1 transduces survival signals in neuronal cells in response to hypoxia-induced apoptotic insults. J Neurosurg. 2016;124(6):1654-64.

28. Carmeliet $P$, Dor $Y$, Herbert JM, Fukumura D, Brusselmans K, Dewerchin M, Neeman M, Bono F, Abramovitch R, Maxwell P, et al. Role of HIF-1alpha in hypoxia-mediated apoptosis, cell proliferation and tumour angiogenesis. Nature. 1998;394(6692):485-90

29. Li Y, Huang C, Feng $P$, Jiang $Y$, Wang W, Zhou D, Chen L. Aberrant expression of miR-153 is associated with overexpression of hypoxiainducible factor-1alpha in refractory epilepsy. Sci Rep. 2016:6:32091.

30. Liu Y, Nie H, Zhang K, Ma D, Yang G, Zheng Z, Liu K, Yu B, Zhai C, Yang S. A feedback regulatory loop between HIF-1alpha and miR-21 in response to hypoxia in cardiomyocytes. FEBS Lett. 2014;588(17):3137-46. 
31. Chen X, Chen G, Cao X, Zhou Y, Yang T, Wei S. Downregulation of BTG3 in non-small cell lung cancer. Biochem Biophys Res Commun. 2013;437(1):173-8.

32. W-f G, X-f Y, D-f S, Zhao S, Y-p L, H-z S, Takano Y, R-j S, J-s L, H-c Z. The roles of BTG3 expression in gastric cancer: a potential marker for carcinogenesis and a target molecule for gene therapy. Oncotarget. 2015;6(23):19841-67.

33. Ou YH, Chung PH, Hsu FF, Sun TP, Chang WY, Shieh SY. The candidate tumor suppressor BTG3 is a transcriptional target of p53 that inhibits E2F1. EMBO J. 2007;26(17):3968-80.

34. Liu JY, Lu JB, Xu Y. MicroRNA-153 inhibits the proliferation and invasion of human laryngeal squamous cell carcinoma by targeting KLF5. Exp Ther Med. 2016;11(6):2503-8.

35. Zhu N, Gu L, Findley HW, Chen C, Dong JT, Yang L, Zhou M. KLF5 interacts with p53 in regulating survivin expression in acute lymphoblastic leukemia. J Biol Chem. 2006;281(21):14711-8.

36. Srivastava IN, Shperdheja J, Baybis M, Ferguson T, Crino PB. mTOR pathway inhibition prevents neuroinflammation and neuronal death in a mouse model of cerebral palsy. Neurobiol Dis. 2016;85:144-54.

37. Fukuyama Y, Ohta K, Okoshi R, Suehara M, Kizaki H, Nakagawa K. Hypoxia induces expression and activation of AMPK in rat dental pulp cells. J Dent Res. 2007;86(9):903-7.

Ready to submit your research? Choose BMC and benefit from:

- fast, convenient online submission

- thorough peer review by experienced researchers in your field

- rapid publication on acceptance

- support for research data, including large and complex data types

- gold Open Access which fosters wider collaboration and increased citations

- maximum visibility for your research: over $100 \mathrm{M}$ website views per year

At $\mathrm{BMC}$, research is always in progress.

Learn more biomedcentral.com/submissions 